\title{
Cosmic-ray protons, nuclei, electrons, and antiparticles under a two-halo scenario of diffusive propagation
}

\author{
Nicola Tomassetti \\ LPSC, Université Grenoble-Alpes, CNRS/IN2P3, \\ F-38026 Grenoble, France; email: nicola.tomassetti@lpsc.in2p3.fr
}

(Dated: September 2015)

\begin{abstract}
We report calculations of cosmic-ray proton, nuclei, antiproton, electron and positron energy spectra within a "two-halo model" of diffusive transport. The two halos represent a simple, physically consistent generalization of the standard diffusion models, which assume a unique type of diffusion for cosmic rays in the whole Galactic halo. We believe instead that cosmic rays may experience a smaller energy dependence of diffusion when they are in proximity of the Galactic disk. Our scenario is supported by recent observations of cosmic ray protons, nuclei, anisotropy, and gamma-rays. We predict remarkably hard antiparticle spectra at high energy. In particular, at $E \gtrsim 10 \mathrm{GeV}$, the antiproton/proton ratio is expected to flatten, while the positron fraction is found to increase with energy. We discuss the implications for cosmic-ray physics and dark matter searches via antimatter.
\end{abstract}

PACS numbers: 98.70.Sa,95.35.+d

Introduction - The observed spectrum of Galactic cosmic rays (CRs) is believed to arise from a combination of two basic plasma astrophysics phenomena: diffusive shock acceleration occurring in supernova remnants, and diffusive transport off magnetic turbulence [1-4]. The paradigm of $\mathrm{CR}$ transport as a particle diffusion process has been successful for decades in explaining the observed high-degree of isotropy in CR arrival directions, and the abundance of secondary Li-Be-B elements arising from $\mathrm{CR}$ collisions with the interstellar matter. The so-called standard models based on this understanding employ several simplifying assumptions such as homogeneity, isotropy, stationarity, and linearity. The traditional description is that CRs are confined in a cylindrical halo that encompasses the Galactic plane, after being accelerated by supernova remnants to power-law spectra in rigidity $\mathcal{R}$ (momentum/charge ratio), $Q \propto \mathcal{R}^{-\nu}$. The diffusion coefficient is assumed to have a rigidity dependence $K \propto \mathcal{R}^{\delta}$ in the whole halo. The combination of acceleration and transport leads to power-law equilibrium spectra $\phi_{p} \sim Q / K \propto \mathcal{R}^{-\delta-\nu}$ for primary components (e.g., protons, $\mathrm{He}, \mathrm{C}, \mathrm{O}, \mathrm{Fe}$ ), while secondary to primary ratios (e.g., $\mathrm{B} / \mathrm{C}, \mathrm{Li} / \mathrm{O}, \mathrm{Ti} / \mathrm{Fe}$ ) scale as $\phi_{s} / \phi_{p} \sim \mathcal{R}^{-\delta}$. The data on primary nuclei and on the $\mathrm{B} / \mathrm{C}$ ratio constrain the parameters $\delta \approx 0.3-0.7$ and $\nu \approx 2-2.4$.

New generation experiments, and in particular the Alpha Magnetic Spectrometer (AMS), are now probing the fine structure of $\mathrm{CR}$ phenomenology. The rapid rise in the positron fraction $\mathrm{e}^{+} /\left(\mathrm{e}^{-}+\mathrm{e}^{+}\right)$at $E \sim 10-300 \mathrm{GeV}$ of energy $[5,6]$, in contrast to the standard expectations $\sim E^{-\delta}$, may suggest the existence of extra sources of high-energy $e^{ \pm}$, including dark matter particle annihilation/decay or known astrophysical sources, but these scenarios demand a reliable understanding the secondary $e^{ \pm}$ production and their propagation [7-12]. The remarkable spectral hardening observed in CR protons and nuclei at $\mathcal{R} \sim 300 \mathrm{GV}$ [13-16] may be a signature of new astrophysical phenomena, occurring either in acceleration or in propagation, that are unaccounted by standard models
[17-21]. Revisiting propagation, as distinct from acceleration, has also interesting implications for the so-called "anisotropy problem" [22]. The anisotropy observations suggest a rather small rigidity dependence for the diffusion at multi-TeV energies $(\delta \approx 0.15)$, which is in contrast with standard model extrapolations based on the $\mathrm{B} / \mathrm{C}$ ratio $(\delta \approx 0.3-0.7)$. Yet a change of diffusion may also be hinted by a high-energy flattening of secondary to primary ratios $[19,22,23]$. Very recently, new $\bar{p} / p$ data presented by AMS have generated widespread interest [24-28], and some authors have pointed out that the interpretation of these data in terms of secondary production demands a slower diffusion of cosmic rays at high energy [29-31]. We also stress that understanding diffusion is crucial, in dark matter searches, for modeling both background and signal of CR antimatter.

In this paper, we report calculations of $\mathrm{CR}$ proton, nuclei, antiproton, electron and positron energy spectra within a numerical implementation of a two-halo scenario of diffusive transport. The idea of having two halos with different diffusion properties represents the simplest but physically consistent generalization of the standard diffusion models. Standard models assume a unique diffusion regime in the whole propagation region, i.e., a factorized rigidity dependence for the function $K$. The increased turbulence in the disk, compared with that at higher latitudes, leads us to believe that there is a spatial change in the CR diffusion properties. This idea was proposed in connection with the detection of a break in the proton and He spectra [18], but the model was unable to describe the sharp structures reported by the PAMELA experiment. The AMS Collaboration has now measured the detailed variations of the proton and He fluxes at $\mathrm{GeV}-$ $\mathrm{TeV}$ energies, showing a significantly smoother spectral hardening. In particular, the proton spectral index is found to progressively increase at $E \gtrsim 100 \mathrm{GeV}$ [16]. As we will show, a two-halo scenario of CR diffusion is supported by these data, and it allows us to understand several puzzling properties of the CR spectrum. Through- 
out this paper, we discuss important consequences for the physical observables that are being investigated by AMS, including implications for dark matter searches via antimatter.

Numerical Implementation - The CR diffusion is described by a $2 \mathrm{D}$ transport equation with boundary conditions in a cylindrical region for all $\mathrm{CR}$ species:

$$
\partial_{t} \psi=Q+\vec{\nabla} \cdot(D \vec{\nabla} \psi)-\psi \Gamma+\partial_{E}(\dot{E} \psi),
$$

where $\psi=\psi(E, r, z)$ is the particle number density as a function of energy and space coordinates, $\Gamma=\beta c n \sigma$ is the destruction rate for collisions off gas nuclei, with density $n$, at velocity $\beta c$ and cross section $\sigma$. The source term $Q$ is split into a primary term, $Q_{\text {pri }}$, and a secondary production term $Q_{\mathrm{sec}}=\sum_{\mathrm{j}} \Gamma_{j}^{\mathrm{sp}} \psi_{\mathrm{j}}$, from spallation of heavier $j$-type nuclei with rate $\Gamma_{j}^{\mathrm{sp}}$. The term $\dot{E}=-\frac{d E}{d t}$ describes ionization and Coulomb losses, as well as radiative cooling of $\mathrm{CR}$ leptons. To numerically solve Eq. 1 in steady state conditions $\left(\partial_{t} \psi=0\right)$, we employ the DRAGON package [32]. We introduced a modification of the finite-differencing scheme and of the spatial grid of the code in order to allow for a diffusion coefficient of the form $K(\mathcal{R}, z)=K_{0}\left(\mathcal{R} / \mathcal{R}_{0}\right)^{\delta(z)}$. Note that our function $K$ is non-separable in rigidity and space coordinates. The full propagation region has half-height $L=4 \mathrm{kpc}$ and radius $r_{\max }=20 \mathrm{kpc}$. We split the cylinder into two $z$-symmetric propagation zones. We call inner halo the region which surrounds the disk for a few hundred pc $\left(|z|<l_{i} \cong 500 \mathrm{pc}\right)$, where the turbulence is presumably injected by SN bubbles, while the outer halo represents a wider region $\left(l_{i}<|z|<L\right)$ possibly characterized by a CR-driven turbulence spectrum [34]. The spatial dependence of $\delta(z)$ is expressed by $\delta=\delta_{i} \cong 0.15$ in the inner halo and $\delta=\delta_{o} \cong 0.75$ in the outer halo, where $K_{0} \cong 10^{28} \mathrm{~cm}^{2} \mathrm{~s}$, and $\mathcal{R}_{0} \equiv 0.25 \mathrm{GV}$ set the diffusion in the two zones. The source spectra are of the type $Q^{\text {pri }} \propto \mathcal{R}^{-\nu}$, with $\nu=2.32$ for nuclei, while proton spectra are steeper by 0.08 [33]. For illustrative purposes, we also set up a standard model of Iroshnikov-Kraichnan type diffusion, with $\delta \equiv 0.5$ everywhere and source spectra with $\nu=2.3$, to match the data at $E \sim 1-200 \mathrm{GeV} /$ nucleon. The local interstellar flux is $\phi=\frac{\beta c}{4 \pi} \psi_{\odot}$, computed at the position $z_{\odot}=0$ and $r_{\odot}=8.3 \mathrm{kpc}$. In the following, the particle fluxes at Earth from the two models are presented. The solar modulation is described under the force-field approximation [35], using modulation parameter values $\Phi \cong 550 \mathrm{MV}$ for protons and leptons (relevant for the AMS data) and $\Phi \cong 350 \mathrm{MV}$ for nuclei (relevant for the PAMELA data) [36, 37].

Protons - The calculated proton spectrum is shown in Fig. 1 in comparison with the new AMS data. At $E \gtrsim 10 \mathrm{GeV}, \mathrm{CR}$ protons are not very sensitive to energy losses or destruction. Our numerical results nicely confirm the trends obtained in our analytical derivations [18]. Neglecting interactions, one can see that the naive expectation, $\phi_{p} \sim Q^{\text {pri }} / \Omega$ with $\Omega=K / L$, has to be modified using $\Omega=\left(l_{i} / k_{i}+l_{o} / k_{o}\right)$, which depends on the
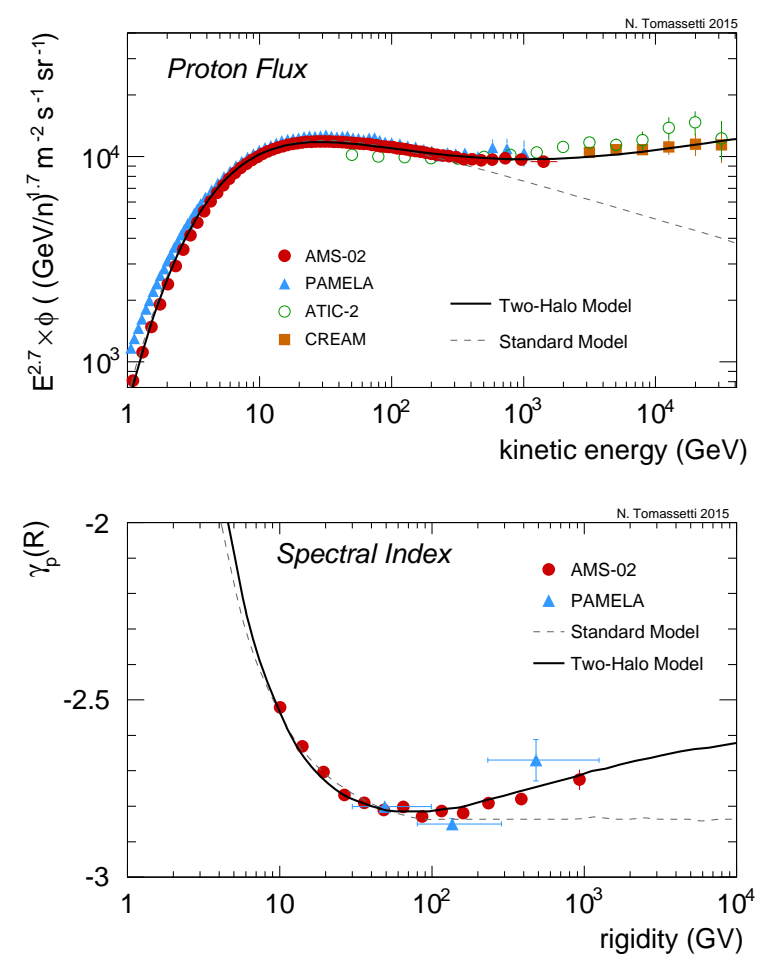

FIG. 1. Top: proton energy spectrum multiplied by $E^{2.7}$. Bottom: rigidity dependence of the proton spectral index. The model calculations are shown in comparison with the data [13-16]. The modulation potential is $\Phi=550 \mathrm{MV}$.

diffusion coefficients $k_{i / o} \propto \mathcal{R}^{\delta_{i / o}}$ and on the half-sizes of the two halos $l_{i / o}$. The CR propagation in both regions, with systematically slower diffusion in the inner halo, is at the origin of the spectral hardening. The high-energy propagation becomes progressively dominated by the inner halo. In the bottom panel, we plot the spectral index $\gamma(\mathcal{R})=d\left[\log \left(\phi_{p}\right)\right] / d[\log (\mathcal{R})]$. Our model describes very well the smooth evolution of the spectrum measured by AMS at $\mathcal{R} \sim 10-1000 \mathrm{GV}$ We also note that, in this rigidity region, the spectrum is never power-law.

Nuclei - The transport of primary nuclei (e.g., He, C, $\mathrm{O}$ or $\mathrm{Fe}$ ) is similar to that of protons, hence we expect a spectral hardening at the same rigidity as for proton. A minor difference comes from destructive interactions, increasing with increasing masses, that slightly harden the spectra below $\sim 100 \mathrm{GeV} /$ nucleon. The carbon spectrum is shown in Fig. 2. At $E \gg \mathrm{GeV} / \mathrm{n}$, its approximate behavior is $\phi \sim Q /(\Omega+h \Gamma)$, where $h \Gamma$ is the interaction term for a surface gas density $h n \cong 200 \mathrm{pc} \times 1 \mathrm{~cm}^{-3}$, acting in competition with the diffusive term $\Omega$. Secondary nuclei, like boron in the figure, are expected to have steeper spectra and to experience a stronger upturn in comparison to their progenitors $\mathrm{C}-\mathrm{N}-\mathrm{O}$. As a consequence the $\mathrm{B} / \mathrm{C}$ ratio is found to progressively flatten, as shown in the figure, to an asymptotic multi- $\mathrm{TeV}$ dependence $\sim E^{-\delta_{i}}$. At lower energies, the $\mathrm{B} / \mathrm{C}$ ratio from our model recovers the standard model behavior. A hint 


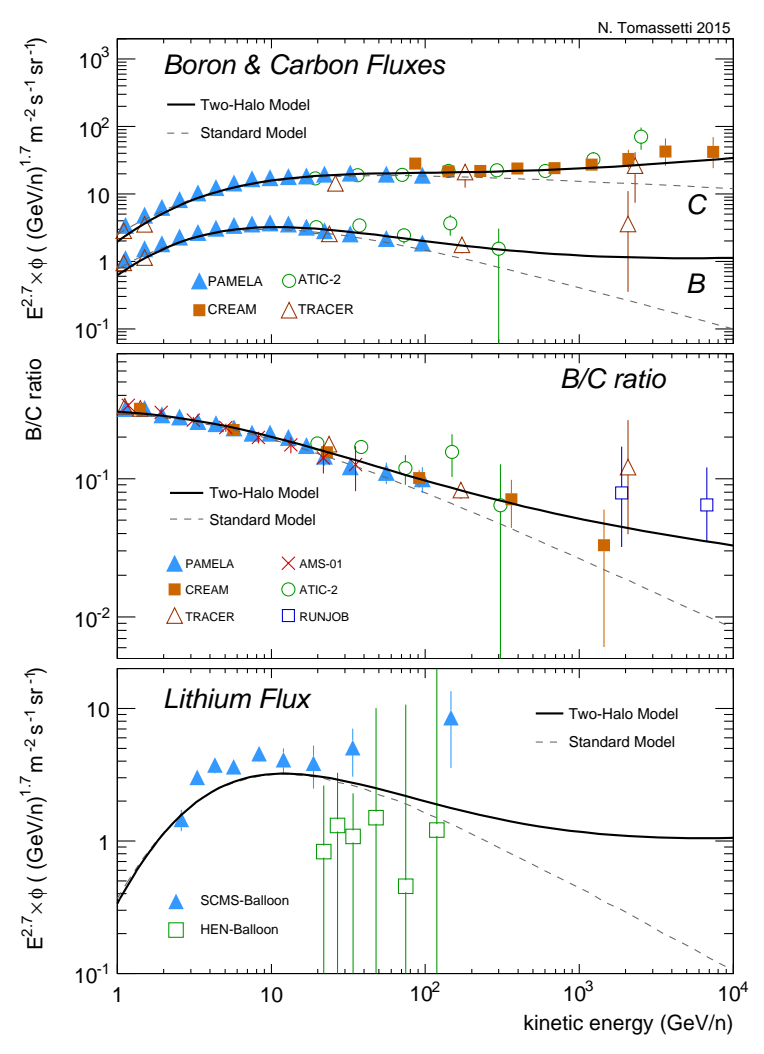

FIG. 2. Top to bottom: Energy spectra of B and C, B/C ratio, and $\mathrm{Li}$ spectrum. The spectra are multiplied by $E^{2.7}$. The model calculations are shown in comparison with the data $[15,38-44]$. The modulation potential is $\Phi=350 \mathrm{MV}$.

of flattening in the $\mathrm{B} / \mathrm{C}$ data is noted in several studies $[11,19,22,23,45]$. The lithium flux, shown in Fig 2, is particularly sensitive to $\mathrm{CR}$ propagation. Its production depends not only on C-N-O collisions, but also on tertiary interactions $\mathrm{B} \rightarrow \mathrm{Li}$ and $\mathrm{Be} \rightarrow \mathrm{Li}$. In spite of the scarcity of present data, the Li flux is one of the best observables for AMS in terms of detection performance, and it is currently being measured to a $\sim 1 \%$ precision up to $\mathrm{TeV} /$ nucleon energies [24]. These data will be very valuable for testing our scenario.

Antiprotons - In contrast to Li-Be-B nuclei, that are ejected with the same kinetic energy per nucleon as their progenitors, the antiprotons emitted in p-p or p-He collisions have broad energy distributions and large inelasticity factors. This type of kinematics reshapes significantly the antiproton production spectrum $Q_{\bar{p}}^{\text {sec }}$. Their subsequent propagation is similar to that of protons, except for the presence of the so-called tertiary $\bar{p}-p$ processes. In Fig. 3 the $\bar{p} / p$ ratio is shown for the two considered models. In the standard model, it decreases smoothly above $\sim 10 \mathrm{GeV}$. In our two-halo model, the ratio has a substantial flattening at $E \sim 10 \mathrm{GeV}-$ a few $100 \mathrm{GeV}$. With the present data, it appears that no room is left for exotic antiproton components. Recent AMS data have triggered exciting speculations about a possible dark matter

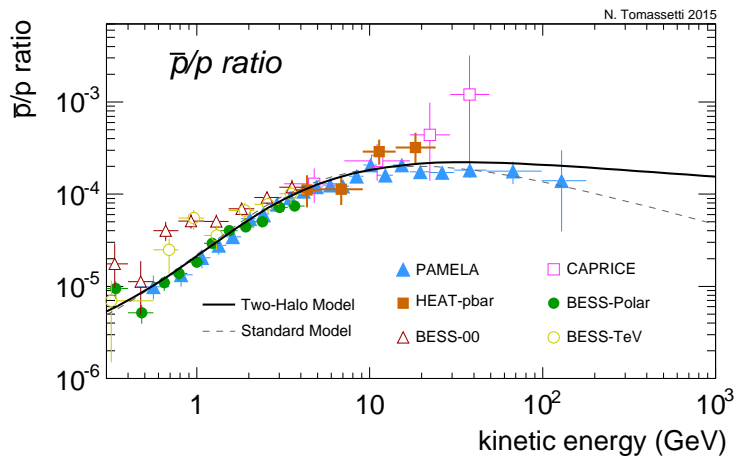

FIG. 3. The $\bar{p} / p$ ratio as function of kinetic energy. The model calculations are shown in comparison with the data [46-51]. The modulation potential is $\Phi=350 \mathrm{MV}$.

induced antiproton excess [25-27]. A flattening of the $\bar{p} / p$ ratio is an unavoidable feature of our scenario and it should be considered when setting upper limits to the search for new-physics signals.

Anisotropy - The CR anisotropy has received renewed attention in recent years $[22,52,53]$. From observations, the dipole anisotropy in the $\mathrm{TeV}$ band remains nearly constant to $\eta \sim 10^{-3}$. Standard model predictions exceed the observational limits by one order of magnitude. To match the data, a diffusion coefficient with $\delta \approx 0.15$ is required. This is indeed the case for the model considered here, which gives $\eta \lesssim 2 \times 10^{-3}$ at $1-100 \mathrm{TeV}$. These estimates depend on the standard formula for anisotropy, $\eta \equiv \frac{3 K}{c} \frac{\nabla \psi}{\psi}$, within the diffusion approximation. We also stress that accounting for the discreteness of the CR sources may induce large deviations from these estimates.

Connections with $\gamma$-ray physics - Although we have focused on local observables, our model predicts also a $\mathrm{CR}$ spectral steepening with increasing distance from the disk. Moreover, one may also argue for a smooth radial dependence for the parameter $\delta$. Some theoretical arguments are found in [23, 34]. Observationally, a spectral steepening toward high latitudes or longitudes has been found in $\gamma$-rays by the Fermi-LAT observatory [54]. As argued in [55] and very recently shown in [56], the FermiLAT data are suggestive of a spatial change of CR diffusion. These works are nicely complementary to the present paper.

Leptons - In contrast to hadrons, light CR leptons are subjected to synchrotron radiation and inverse Compton losses with a characteristic time-scale $\tau(E) \propto E^{-1}$. These processes limit the range that they can travel to distance scales $\lambda_{i / o} \sim \sqrt{\tau k_{i / o}}$, in the two propagation regions. Above a few $\mathrm{GeV}$ one has $\lambda_{o} \lesssim l_{o}$ in the outer halo so that, in contrast to hadrons, CR leptons detected at Earth have spent a larger fraction of time in the inner halo. At higher energies the distance scale decreases further. The resulting effect is that the $e^{ \pm}$spectra above a few tens of $\mathrm{GeV}$ are essentially determined 


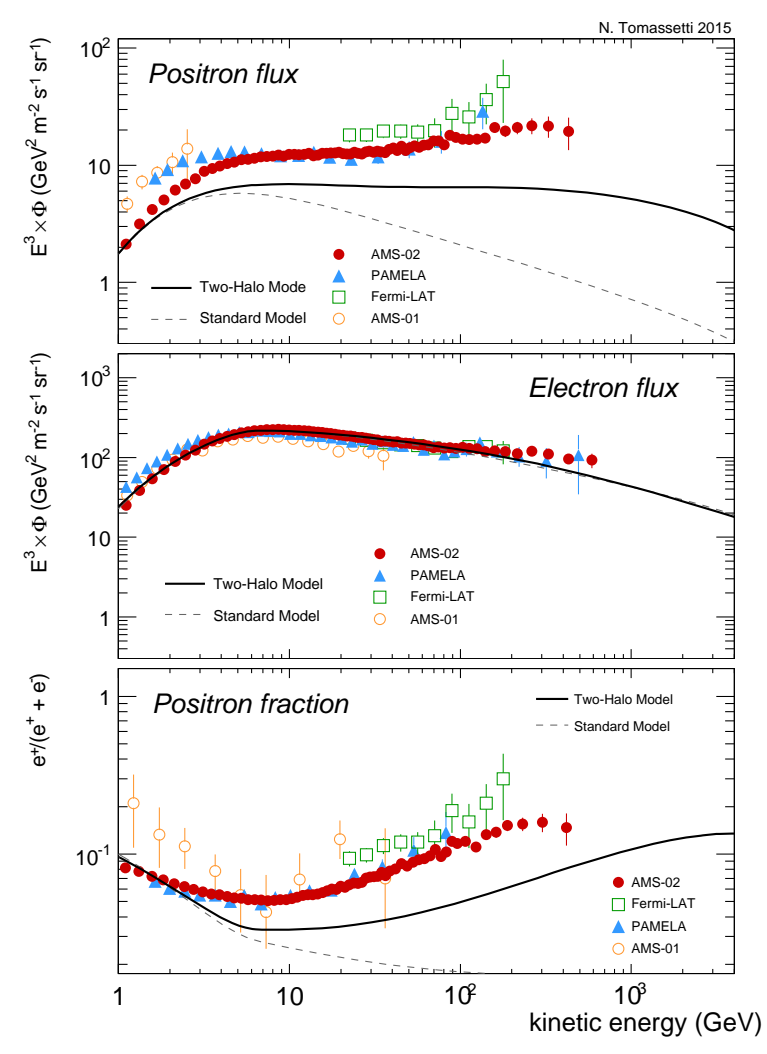

FIG. 4. Energy spectra of positrons and electrons multiplied by $E^{3}$, and positron fraction $\mathrm{e}^{+} /\left(\mathrm{e}^{-}+\mathrm{e}^{+}\right)$. The model calculations are shown in comparison with the data [5, 6, 59-62].

by the propagation in the inner halo. Radiative losses also steepen the $e^{ \pm}$spectrum with a stronger effect than diffusion. The lepton fluxes at $E \gtrsim 10 \mathrm{GeV}$ are of the type $\phi_{e^{ \pm}} \sim(\tau / K)^{1 / 2} Q$ and their spectral index scales as $\gamma_{e} \approx-\nu_{e}-\frac{1}{2}(\delta+1)$ where, for the two-halo scenario, we have $K \cong k_{i}$ and $\delta \cong \delta_{i}$. In summary, the leptonic spectra under the two-halo model are expected to be harder, due to propagation, by a factor $\frac{1}{2}\left(\delta-\delta_{i}\right) \approx 0.15-0.2$. In addition, secondary $e^{ \pm}$have a further hardening factor coming from the spectra of their parent nuclei p-He. The results of our numerical calculations are shown in Fig. 4. The force-field approximation is inadequate for CR leptons [57], hence we focus on $E \gg \mathrm{GeV}$. It can be seen that our model gives an enhanced flux of secondary positrons at high energy. For primary electrons we expect a similar effect, but their flux depends on the adopted injection index $\nu_{e}$, which is not known a priori. Using the quantity $\Delta \Phi_{e^{ \pm}} \equiv \phi_{e^{-}}-\phi_{e^{+}}$to minimize the secondary and extra components [58], we derived $\nu_{e} \cong 2.6$ for the standard model and $\nu_{e} \cong 2.77$ for the two-halo model. Hence the propagated electron fluxes for the two models are similar. It is interesting to note that our model, with the adopted parameters, matches the conditions for having a positron fraction that increases with energy.

As for the case of antiprotons, a high-energy enhancement of the positron flux is a characteristic feature of the two-halo scenario of CR diffusion. The high-energy positron flux is significantly larger than that arising from standard models. For dark matter models with $\mathrm{TeV}$ scale masses, the increased level of background, together with a much slower propagation of the signal, will changes the intensity and the spectral shape of the expected $e^{ \pm}$yield from exotic sources. At this point, one may be tempted to explain the $e^{ \pm}$data entirely with a purely diffusive picture. However, we found that even the extreme choice $\delta_{i} \cong 0$ is unsatisfactory for CR leptons. Besides, this would require exceedingly steep injection spectra and a worse agreement with the $\mathrm{B} / \mathrm{C}$ ratio data. Our choice of $\delta_{i} \cong 0.15$ represents at present the best agreement between $B / C$ data and anisotropy requirements. Nonetheless, the current $B / C$ data are not very constraining. Light-nuclei data from AMS will allow for much more stringent predictions.

Conclusions - Several observed properties of the CR spectrum can be understood in terms of spatial changes of CR diffusion in the Galaxy, in contrast to standard propagation models that assume a unique diffusion regime everywhere. In our scenario, a departure from the standard power-law form is predicted for all elemental fluxes. We found an excellent agreement with the new AMS proton data at $\sim 1 \mathrm{GeV}-2 \mathrm{TeV}$ of energy. Our model can reconcile the steeply falling low-energy shape of the $\mathrm{B} / \mathrm{C}$ ratio with the high-energy requirements of the anisotropy data. In this we have devised perhaps one of the few viable solutions for the anisotropy problem. Our model shares some consequences with the model proposed in [19]. The $\gamma$-ray diffuse emission may be useful to discriminate the two scenarios. Our predictions have also implications for the Galactic synchrotron emission, which traces the electron spectrum at different latitudes [63]. Interesting results are found for the antiparticle spectra, especially in view of recent excitement about possible evidence for dark matter. Our model predicts remarkably hard spectra for secondary antiproton and positron at high energy. In particular, the $\bar{p} / p$ ratio is found to flatten above $\sim 10 \mathrm{GeV}$, while the positron fraction is found to increase with energy. These results have a direct impact on upper limits on new physics signals using the $\bar{p} / p$ ratio, or on dark matter models for the positron fraction. The antimatter spectra are strictly related to secondary nuclei. The main limitation is represented by the large uncertainties of the pre-AMS nuclear data. A more robust assessment will be done upon the forthcoming release of the AMS data on $\mathrm{He} \mathrm{Li}, \mathrm{C}, \mathrm{B} / \mathrm{C}$ and $\bar{p} / p$. In particular, the Li flux will be a powerful tool at the level of precision expected by AMS. If new data confirms our scenario, then the current standard models of CR diffusion will have to be revised to account for it.

I thank Jin Chao and Carmelo Evoli for discussions and support with DRAGON, Sherry Chou for reading this manuscript, and the DRAGON team for sharing their code with the community. Several data are extracted from the CRDB database [36]. This work is supported by the ANR LabEx grant ENIGMASS at CNRS/IN2P3. 
[1] I. A. Grenier, J. H. Black, A. W. Strong, Annu. Rev. Astron. Astrophys., 53, 199-246 (2015);

[2] T. Bell, Braz. J. Phys., 44, 415 (2014);

[3] P. Blasi, Astron.Astrophys.Rev., 21, 70 (2013);

[4] A. W. Strong, I. V. Moskalenko, V. S. Ptuskin, Ann. Rev. Nucl. Part.Sci., 57, 285-327 (2007);

[5] L. Accardo et al. (AMS-02), PRL, 113, 121101 (2014);

[6] O. Adriani et al. (PAMELA) Nature, 458, 607 (2009);

[7] K. Y. Choi, B. Kyae, C. S. Shin, PRD, 89, 055002 (2014);

[8] K. Blum, B. Katz, E. Waxman, PRL, 111, 211101 (2013);

[9] P. D. Serpico, Astropart. Phys., 39-40, 2 (2012);

[10] S. J. Lin, Q. Yuan, X. J. Bi, PRD, 91, 063508 (2015);

[11] P. Mertsch \& S Sarkar, PRD, 90, 061301 (2014);

[12] N. Tomassetti \& F. Donato, ApJ, 803, L15 (2015);

[13] O. Adriani et al. (PAMELA), Science, 332, 69 (2011);

[14] H. S. Yoon et al. (CREAM), ApJ, 728, 122 (2011);

[15] A. D. Panov et al. (ATIC-2) Bull. Russian Acad. Sci. 73-5, 564 (2009);

[16] M. Aguilar et al. (AMS-02), PRL, 114, 171103 (2015);

[17] V. Ptuskin, V. Zirakashvili, E. S. Seo, ApJ, 763, 47 (2013);

[18] N. Tomassetti, ApJ, 752, L13 (2012);

[19] P. Blasi, E. Amato, P. D. Serpico, PRL 109, 061101 (2012); see also R. Aloisio et al., arXiv:1507.00594 (2015);

[20] A. E. Vladimirov et al., ApJ, 752, 68 (2012);

[21] S. Thoudam \& J. Horandel, A\&A, 567, A33 (2014);

[22] M. Pohl \& D. Eichler, ApJ, 766, 4 (2013);

[23] C. Evoli \& H. Yan, ApJ, 782, 36 (2014);

[24] AMS Collaboration, AMS Days at CERN (2015) - These data are not used in the present work. Given their preliminary nature, we will wait for their publication.

[25] C. H. Chen, C. W. Chiang, T. Nomura, PLB, 747, 495499 (2015);

[26] K. Kohri et al., arXiv:1505.01236 (2015);

[27] K. Hamaguchia, T. Moroia K. Nakayamaa, PLB, 747, 523-528 (2015);

[28] M. Kachelrieß, A. Neronov, D. V. Semikoz, arXiv:1504.06472 (2015);

[29] G. Giesen et al., arXiv:1504.04276 (2015);

[30] R. Kappl, A. Reinert, M. W. Winkler, arXiv:1506.04145 (2015);

[31] R. Cowsik, B. Burch, T. Madziwa-Nussinov, ApJ, 786,
124 (2014), see also arXiv:1505.00305 (2015);

[32] C. Evoli, et al., JCAP 10 (2008) 018;

[33] This is a known issue that we ascribe to injection, see e.g., Malkov et al., PRL 108, 081104 (2012); Y. Ohira, \& K. Yoka, ApJ, 729, L13 (2011);

[34] A. D. Erlykin \& A. Wolfendale, J.Phys.G:NPP,28,2329 (2002);

[35] L. J. Gleeson, \& W. I. Axford, ApJ, 154, 1011 (1968);

[36] D. Maurin, F. Melot, R. Taillet, A\&A, 569, A32 (2014);

[37] D. Maurin et al., Adv.Space Res. 55, 363-389 (2015);

[38] O. Adriani et al. (PAMELA), ApJ, 791, 93 (2014);

[39] H. S. Ahn et al. (CREAM), 714, L89 (2010);

[40] A. Obermeier, P. Boyle, J. Hórandel, D. Múller, ApJ, 752, 69 (2012);

[41] M. Aguilar et al., ApJ, 724, 329 (2010);

[42] V. A. Derbina et al. (RUNJOB), ApJ, 628, L41 (2005);

[43] C. D. Orth et al. (SCMS) ApJ, 226, 1147 (1978);

[44] E. Juliusson (HEN), ApJ, 191, 331 (1974);

[45] N. Tomassetti \& F. Donato, A\&A, 544, A16 (2012);

[46] O. Adriani et al. (PAMELA) PRL, 105, 121101 (2010);

[47] A. S. Beach et al. (HEATPbar), PRL, 87, 271101 (2001);

[48] M. Boezio et al. (CAPRICE) ApJ, 561, 787 (2001);

[49] K. Abe et al. (BESS-Polar), PLB, 670, 103 (2008);

[50] Y. Asaoka et al. (BESS-00), PRL, 88, 051101 (2002);

[51] S. Haino et al. (BESS-TeV) 29 ${ }^{\text {th }}$ ICRC, 3-13 (2005);

[52] V. Savchenko, M. Kachelrieß, D. V. Semikoz, arXiv:1505.02720 (2015);

[53] R. Kumar \& D. Eichler, ApJ, 785, 129 (2014);

[54] M. Ackermann et al., ApJ750, 3 (2012);

[55] A. Erlykin \& A. Wolfendale, Astrop.Phy. 42, 70-75 (2013);

[56] D. Gaggero et al., PRD, 91, 083012 (2015);

[57] L. Maccione PRL, 110, 081101 (2013);

[58] C. Jin, Y. Q. Guo, H. B. Hu, arXiv:1504.06903 (2015);

[59] M. Aguilar et al. (AMS-02), PRL, 113, 121102 (2014);

[60] O. Adriani et al. (PAMELA), PRL, 111, 081102 (2013);

[61] M. Aguilar et al. (AMS-01), PLB, 646, 145 (2007);

[62] A. A. Abdo et al. (Fermi-LAT), PRL, 108, 011103 (2012);

[63] E. Orlando \& A. W. Strong, MNRAS, 436, 2127-2142 (2013); 